\title{
CHANGES IN TKA KINEMATICS AND CONTACT FORCES INDUCED BY MAL-CONFIGURATIONS: A NUMERICAL STUDY
}

\author{
Silvia Pianigiani (1), Luc Labey (2), Walter Pascale (1), Bernardo Innocenti (2) \\ 1. I.R.C.C.S. Istituto Ortopedico Galeazzi, Milano, Italy; 2. European Center for Knee \\ Research, Smith\&Nephew, Leuven, Belgium
}

\section{Introduction}

Total Knee Arthroplasty (TKA) is a very successful surgical procedure, but patients with difficulties or pain during motion still persist. TKA patient outcomes can be affected not only by implant design, but also by implant position and by patientrelated anatomical factors. To check how and how much the influence of these factors can alter both tibio-femoral (TF) kinematics and TF and patellofemoral (PF) contact forces, a numerical analysis was performed on several TKA designs during squatting.

\section{Methods}

Physiological bone models were obtained from a $\mathrm{CT}$ of a cadaver leg and the physiological soft issue insertion points were localized according to literature [Innocenti, 2011; Victor, 2009a]. Four TKA designs were implanted according to their surgical guidelines in the physiological model: a conventional fixed bearing design; a high flex fixed bearing design; a mobile bearing design; a hinge prosthesis design).

The following configurations were analyzed [Innocenti, 2011]: 1. the theoretical configuration, corresponding to the surgical guideline technique, and physiological locations of the soft tissue insertion points; 2. a change in position of the tibial component in medio-lateral (ML) and anteroposterior (AP) directions; 3 . a change in orientation of the tibial component in flexion-extension (FE), abduction-adduction (AA) and internal-external (IE) orientation; 4. patella alta and patella baja; 5. tilting of the patellar component (IE rotation); 6. a change in location of the two collaterals ligaments in ML, AP and proximo-distal (PD) directions.

For each prosthesis and configuration, a squat up to $120^{\circ}$ of flexion, with a vertical hip force of $200 \mathrm{~N}$, was simulated using LifeMOD/KneeSIM 2007.0.5 (LifeModeler, Inc., San Clemente, CA) following the same procedure as described in experimental tests [Victor, 2009b]. TF kinematics along IE, AA and $\mathrm{AP}$ axes and TF and PF maximal contact forces are observed and compared for all the 98 configurations in analysis.

\section{Results}

The frequency with which deviations from the reference kinematics occur due to tibial component malpositioning, patellar malpositioning and the soft-tissues anatomy are listed in Table 1 . We discriminated between three levels of deviation, referring to the reference configuration, in terms of TF kinematics $\left(2^{\circ}, 3^{\circ}\right.$ and $4^{\circ}$ or $2 \mathrm{~mm}, 3 \mathrm{~mm}$ and 4 $\mathrm{mm})$ and contact forces $(10 \%, 20 \%$ and $30 \%)$.

\begin{tabular}{|c|c|c|c|}
\hline & \begin{tabular}{|c|} 
Tibial \\
component
\end{tabular} & $\begin{array}{c}\text { Patellar } \\
\text { component }\end{array}$ & $\begin{array}{l}\text { Collateral } \\
\text { Ilgaments }\end{array}$ \\
\hline Difference in IE iotation $\geq 2^{n}$ & 11 & 1 & 11 \\
\hline Difference in IE rotation $\geq 3^{\circ}$ & 2 & 1 & 6 \\
\hline Difference in IE rotation $\geq 4^{\circ}$ & 1 & 1 & 4 \\
\hline Difference in $M$ tilting $\geq 2^{\circ}$ & 5 & 0 & 1 \\
\hline Difference in $\mathrm{AA}$ tilting $\geq 3^{\circ}$ & 4 & 0 & 0 \\
\hline Difference in $A A$ tilting $>4^{\circ}$ & 1 & ก & n \\
\hline Difference in AP translation $\geq 2 \mathrm{~mm}$ & 10 & 0 & 0 \\
\hline Difference in AP translation $\geq 3 \mathrm{~mm}$ & 2 & 0 & 0 \\
\hline Difference in AP translation $\geq 4 \mathrm{~mm}$ & 0 & 0 & 0 \\
\hline PF contact force $\geq 10 \%$ & 6 & 12 & 0 \\
\hline PF contact force $\geq 20 \%$ & 3 & 6 & 0 \\
\hline PF contact force $\geq 30 \%$ & 2 & 3 & 0 \\
\hline Medial It contact force $\geq 10 \%$ & 1 & 1 & 12 \\
\hline Medial TF contact force $\geq 20 \%$ & 4 & 2 & 9 \\
\hline Medlal TF contact force $\geq \mathbf{3 0 \%}$ & 4 & 0 & 2 \\
\hline Lateral TF contact force $\geq 10 \%$ & 4 & 4 & 14 \\
\hline Lateral TF contact force $\geq 20 \%$ & 1 & 0 & 9 \\
\hline Lateral TF contact force $\geq 30 \%$ & 0 & 0 & 4 \\
\hline
\end{tabular}

Table 1: The table shows the frequency [\%] of affected configurations, with respect to the total number of analyzed mal-configurations.

\section{Discussion}

In this project the effects of tibial mal-positioning, patellar mal-positioning and soft-tissues anatomies on knee kinematics and kinetics were numerically analyzed and correlated for different TKA designs.

The used model has been validated both for the contact force analysis [Innocenti, 2011] and for the kinematics analysis [D’Lima, 2011; Nakamura, 2010; Victor, 2009b]. Deviations in kinematics are usually quite small (smaller than $5^{\circ}$ or $4 \mathrm{~mm}$ ), thus proportional to the applied changes in alignment, while changes in contact forces can reach much higher values [Innocenti, 2011]. The same small changes in configuration which lead to the small changes in kinematics, can cause dramatic differences in contact forces. This finding has important clinical implications: a relatively small surgical errors, which may not be detectable using standard follow-up tools used by clinicians, can still lead to considerable differences in pressures and their distributions, strains in ligaments and, as a consequence, pain.

\section{References}

D'Lima et al, Clin Orthop 469:2953-2970 2011. Innocenti et al, J Biomech, 44:1573-1581, 2011. Nakamura et al, JoA 25:3, 2010

Victor et al, The Knee, 16:358-65, 2009a.

Victor et al, JBJSAm. 91:150-163, 2009b. 